\title{
Study on Lateral Aerodynamic Characteristics of Hypersonic Lifting-Configuration
}

\author{
Qing Gao ${ }^{1}$, Jianhua $\mathrm{Li}^{2}$, Junbo Zhao ${ }^{1}$ and Qian $\mathrm{Li}^{1}$ \\ 1. The Second Department, China Academy of Aerospace Aerodynamic, Beijing 100074, China \\ 2. The Eleventh Department, China Academy of Aerospace Aerodynamic, Beijing 100074, China
}

Received: August 27, 2014 / Accepted: October 10, 2014 / Published: December 31, 2014.

\begin{abstract}
To have a deep understanding of the lateral stability of hypersonic lifting-configurations, wind-tunnel tests of roll static and dynamic stability for typical hypersonic lifting-configurations are carried out. The results show the roll is static unstable in small angles; the roll dynamic test curves present obvious non-linearity characteristics, and the model vibrates violently even when the angle of attack is small, which may be provoked by the non-symmetry transition from the small transverse flow around the nose of model. Subsequent research adopts longitudinal trips to generate symmetry transition at the fore-body of the model. As a result, the lateral stability of the aircrafts is apparently improved. The results show that the lateral stability of hypersonic aircrafts is very weak, and the main reason for this is lateral perturbation of flow over the nose, among which asymmetric transition weighs the most. Adoption of longitudinal trips could spur fixed transition of lateral flow, reduce the transition asymmetry of lateral flow, and strengthen the lateral stability of hypersonic aircrafts at the same time.
\end{abstract}

Key words: Hypersonic lifting-configuration, lateral stability, wind-tunnel test, asymmetric transition.

\section{Introduction}

Hypersonic vehicles promise to be among the greatest technological challenges ever taken. It is expected to have great potential as a low-cost launch vehicle, a high-speed yet economical civilian transport, and a highly responsive military aircraft.

The success of flight test of X-43 in 2004, marked great progress in scramjet, but X-43 only flied $11 \mathrm{sec}$ in hypersonic speed, and the reason may be some problems, such as stability and control have not been well solved. One of the causes for the failures of flight test of HTV-2 (Hypersonic Technology Vehicle 2) in 2010 and 2011 lies in the deficiency of stability and maneuverability during reentry/glide transition stage, yielding roll/yaw coupling movement, introducing yaw/roll moment overshot, far above the practice control ability, leading to losing control of attitude.

Corresponding author: Jianhua Li, senior engineer, research field: aircraft aerodynamic design. E-mail: ssanliren@126.com.
Although RCS (Reaction Control System) system is used for lateral manipulation, the efficiency is limited. So, very possibly, both flight test failures of HTV-2 have close relation with shortage of lateral stability.

For lateral stability of hypersonic vehicles, a lot of computation and wind-tunnel tests have been taken on by the American aerospace community [1-3]. The results show the stability of hypersonic vehicles share characteristics as: weak in lateral stability, severe lateral aerodynamic coupling, sensitive to the influence of air-breathing propulsion, and there is inter-confinement between lateral and longitudinal aerodynamic characteristics.

According to dynamic stability wind-tunnel test of hypersonic vehicles in CAAA (China Academy of Aerospace Aerodynamic), compared with stability in the directional direction, the stability in lateral direction is weaker. In order to deepen understanding of the lateral stability of hypersonic lifting-configuration and the flow around it, wind-tunnel tests are carried out 
on static and dynamic stability characteristics of typical hypersonic lifting-configuration.

\section{Design of Wind-Tunnel Test Model}

The hypersonic lifting-configuration studied in this paper is based on the open configuration data of HTV-2, with typical characteristics, such as plane-symmetry, small control surfaces of this kind of vehicles. The wind-tunnel test results of this model can describe the lateral aerodynamic characteristics of HTV-2 like aircrafts.

The test is carried out in CAAA FD-07. A 0.12-scale model is designed, whose length, span and blockage ratio satisfy requirement of hypersonic wind-tunnel test. For the reason that the test model is comparatively large, to ease loading on the balance system, the model is made of aluminium, assuring the intensity and rigidity, the internal of the model is excavated to almost the maximum extent (see Fig. 1).

\section{Wind-Tunnel Test on Roll Static Stability}

Roll static stability wind-tunnel test of Ma (Mach) $=$ 5 is carried out of HTV-2 like model. The Reynolds number per unit length $R e / L=2.3 \times 10^{7}$. The aerodynamical balance used in the test is TG624C. The maximum uncertainty of the balance is $0.5 \%$ (see Fig. 2).

Fig. 3 gives the roll static stability derivative test results, in which, when the attack angle is below $10^{\circ}$, the model is static unstable, the reason for this may be the model is like low wing, compared with high wing and aircraft with wing at the center line, and low wing downgrades the roll static stability of aircraft. In the

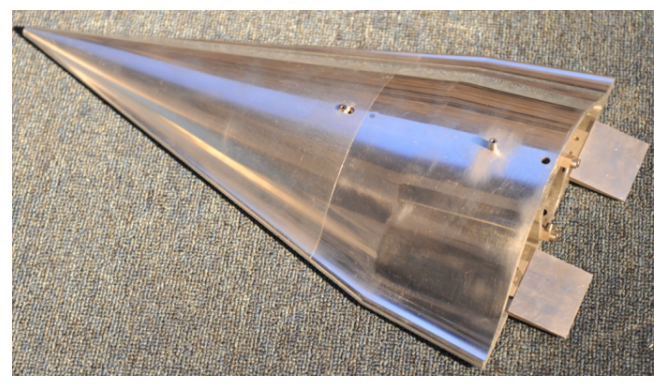

Fig. 1 The wind-tunnel test model.

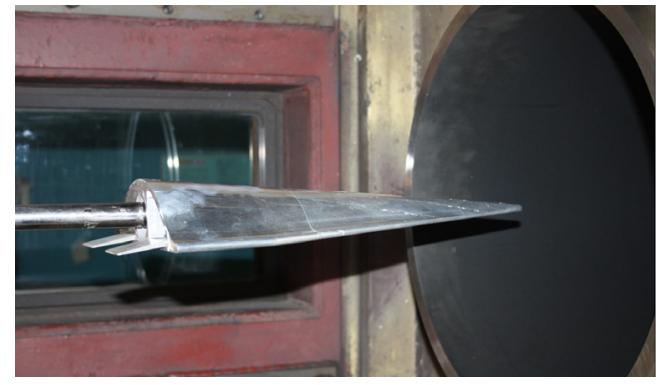

Fig. 2 Model in wind-tunnel.

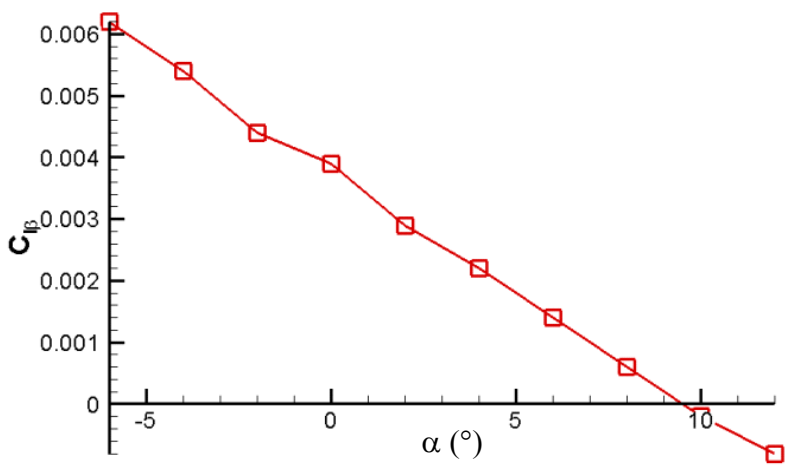

Fig. 3 Variation of roll moment with angle of sideslip $C_{1}$ with angle of attack.

attack angle domain studied, with the increase of the attack angle, the roll static stability is enhanced, because the roll static stability is proportional to lift force and the angle of attack.

In a word, there are some problems in the roll static stability of this aircraft, the roll is static instable in small angle of attack.

\section{Wind-Tunnel Test on Roll Dynamic Stability}

The roll dynamic stability test adopts free-oscillation method. The roll free-oscillation test apparatus is composed of elastic pivot, suspension system, exciting system, data acquisition and procession system. The rigidity of pivot is $12.5 \mathrm{~N} . \mathrm{m} / \mathrm{rad}(\mathrm{N}$ denotes force, $\mathrm{m}$ denotes length, meter, rad denotes angle), the free oscillation frequency of the mechanical model system is $10.6 \mathrm{~Hz}$.

As shown in Fig. 4, the model is linked to the supporting rod through elastic pivot, center of which is coincidence with mass center of model. The model can oscillate freely in roll direction around the center line 


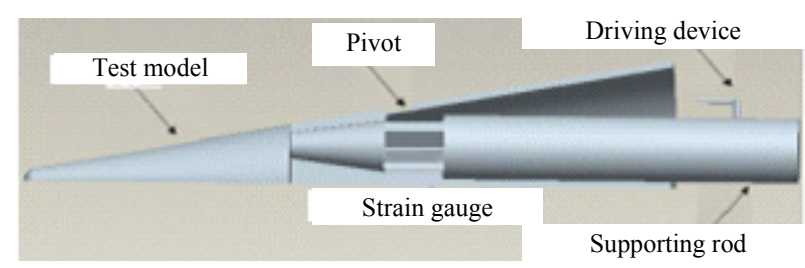

Fig. 4 The roll free-oscillation test apparatus.

of the model. When tested, first, the wind-tunnel is started, then, the model is pushed by the triggering device at the rear of the model, and deviated from the equilibrium position, introducing an initial angular misalignment $\varphi_{0}$ (usually $\varphi_{0}= \pm 1^{\circ}- \pm 3^{\circ}$ ), after triggering device leaves, the model will roll oscillation periodically. Under the coaction of elastic force and aerodynamic force, the amplitude of oscillation will decay with time. There is strain gauge on pivot measuring real-time deformation which is later transmitted to variation of angular misalignment of model.

Seen from Fig. 5, the roll test curves of hypersonic lifting-model present certain multi-frequency and periodical characteristics. The spectrum analysis of roll vibration data under several different flow conditions shows that they share three main vibration frequencies, apart from the mechanical vibration frequency, which indicated that there are three characteristic lengths in the hypersonic lifting-configurations flow field, that is, there are three scales of lateral separation or transition. That is caused by the unique characteristics of flow around the hypersonic aircrafts. Different from the flow around vehicles in subsonic, transonic and supersonic, in which the large scale flow structures, such as shock wave and separation, are in the majority, and the small scale flow structures, such as turbulence and transition, are in the minority; while, in hypersonic flow field, with the increase of small scale and high frequency perturbation, and with the augment of the Mach number, the flow energy which is originally mainly in the longitudinal direction, is partly transmitted to the lateral direction. The result is that the flow field is not donated by a single flow factor but is under the coaction of several different flow factors. Multi-scale is one of the most significant characteristics of hypersonic flow field.
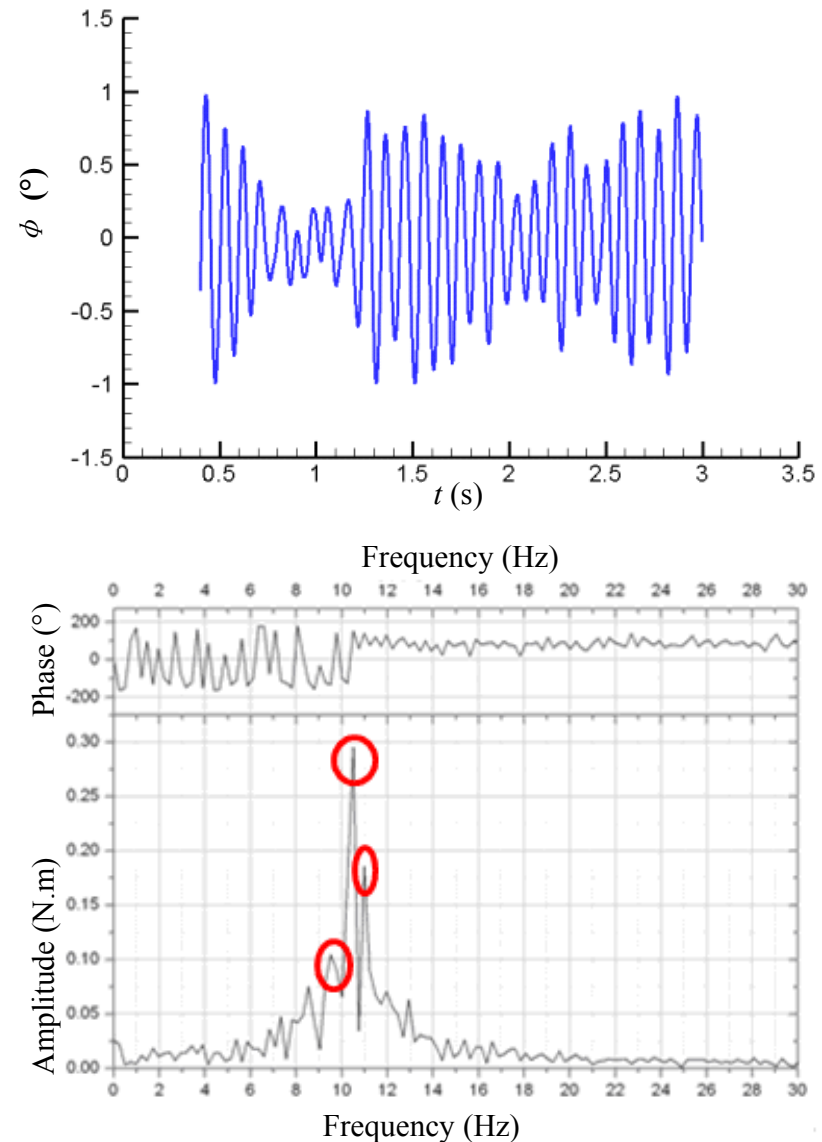

Fig. 5 The roll free oscillation test curve and its spectrum analysis of $\mathrm{Ma}=5, \alpha=\beta=0^{\circ}$.

\section{Measures for Enhancing Lateral Stability}

The nonlinearity and multi-frequencies of roll oscillation curve make hypersonic flight and control very difficult. The aerodynamic measures for enhancing lateral stability should aim at controlling the multi-scale flow structure. According to the researches carried out in CAAA [4], the multi-scale flow structure may be triggered by the non-symmetry transition caused by small lateral flow perturbation around the nose, which will excite the flow non-symmetry downstream, forming lateral circulation flow and lateral non-symmetry aerodynamic force, and deteriorate the lateral stability of hypersonic vehicles. For the reasons above, this paper carries out wind-tunnel tests to check the aerodynamic measures of controlling the flow transition of the nose. For the reason that with the reducing of flight altitude, transition is inevitable, the method of rejecting 
transition is infeasible. On the other hand, the method of spurring symmetry transition on the nose by the trips may be effective.

Wind-tunnel tests are carried out to study the effects of pasting longitudinal trips on the fore-body of the model on lateral stability. Nylon trips of 1-mm high are bended on the above and below of the fore-body (Fig. $6)$.

The wind-tunnel tests results are shown by pasting longitudinal trips along the free stream on the fore-body of the model, forcing symmetry transition at the trips, and they greatly improve the linearity of the test curves, and increase the roll stability as well (Fig. 7), which indicate: (1) the lateral perturbation comes from the transition effects from the flow around the nose; (2) longitudinal trips can improve the symmetry of transition and increase lateral stability of the vehicle.

The reason why adoption of longitudinal trips along the free stream on the fore-body of the model could strength the lateral stability is that altering the separation and transition point on the fore-body will change the flow structure of hypersonic vehicle radically, influencing the attitude stability and controllability straightforward.

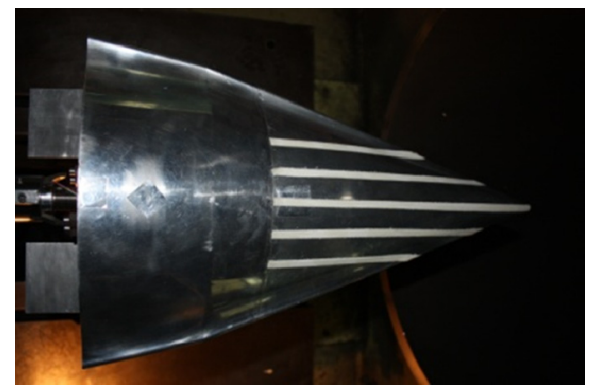

(a)

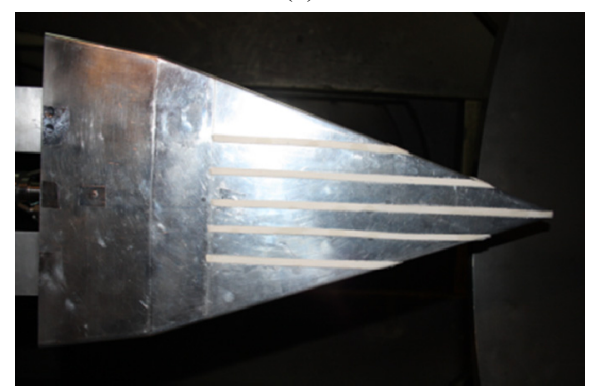

(b)

Fig. 6 Bended trips above (a) and below (b) of the model.

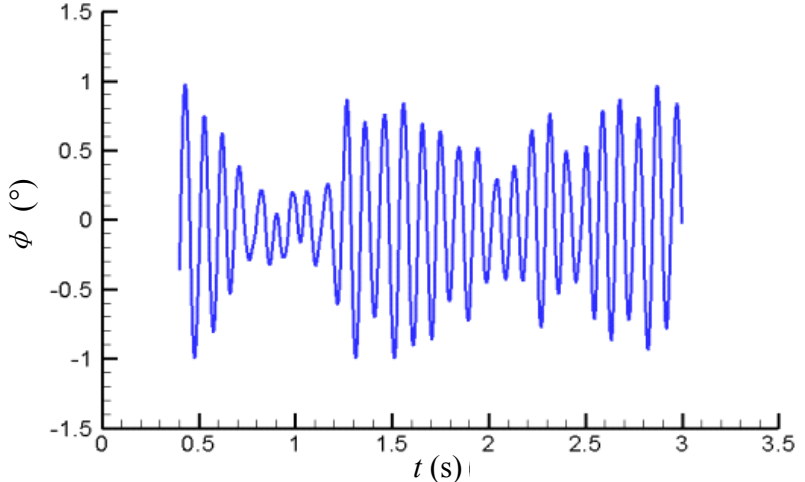

(a)

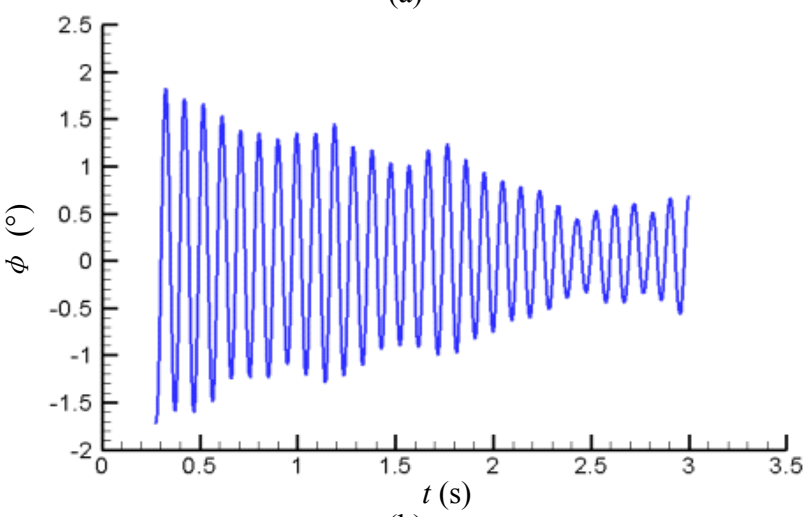

(b)

Fig. 7 The roll free oscillation test lines of model of before (a) and after (b) pasting trips of states: $\mathrm{Ma}=5, \alpha=\beta=0^{\circ}$.

\section{Conclusion}

Wind-tunnel test of roll static and dynamic stability for typical hypersonic lifting-configuration is carried out. The results show that for the reason of the layout of configuration, the model is roll static unstable in small angle of attack. The roll test curves of $\mathrm{Ma}=5$ of hypersonic lifting-model present a certain multi-frequency and periodical characteristics. The results of spectrum analysis of roll vibration data under several different flow conditions show they share three main vibration frequencies apart from the mechanical vibration frequency, which indicates that there are three characteristic lengths in the hypersonic lifting-configuration flow field, that is, there are three scales of lateral separation or transition. Multi-scale is one of the most significant characteristics of hypersonic flow field.

Analysis show the multi-scale lateral perturbation is mainly caused by the non-symmetry transition around the nose of model. By pasting longitudinal trips along 
the free stream on the nose of the model, the non-symmetry in the flow field is weakened, and the lateral stability of hypersonic lifting-configurations is enhanced. The wind-tunnel tests results indicate: (1) the lateral perturbation comes from the transition effects from the flow around the nose; (2) longitudinal trips can improve the symmetry of transition and increase lateral stability of the vehicle.

\section{Reference}

[1] Penland, J. A., Dillon, J. L., and Pittman, J. L. 1978. “An
Aerodynamic Analysis of Several Hypersonic Research Airplane Concepts from $\mathrm{M}=0.2$ to 6.0." Journal of Aircraft 15 (11): 716-23.

[2] Phillips, W. P., and Cruz, C. L. 1993. "Hypersonic Aerodynamic Characteristics for Langley Test Technique Demonstrator.” AIAA 93-3443, NASA Langley Research Center Hampton, Virginia, USA.

[3] Hayes, C. 1981. "Aerodynamic Characteristics of a Series of Airbreathing Missile Configurations.” NASA Langley Research Center, ADA-111786.

[4] Qing, G., and Qian, L. 2012. "The Influence of Asymmetric Transition on Stability of Hypersonic Aircrafts." Tactical Missile Technology 6: 12-15. 